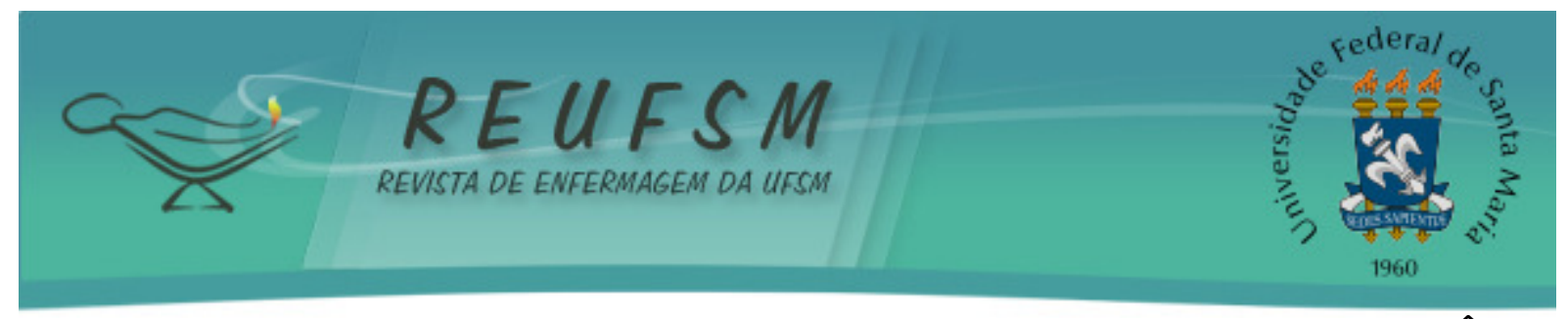

RELATO DE EXPERIÊNCIA

\title{
ACOLHIMENTO COM CLASSIFICAÇÃO DE RISCO NA UNIDADE DE PRONTO ATENDIMENTO: UM RELATO DE EXPERIÊNCIA
}

\author{
RECEPTION WITH RISK RATING IN READY CARE UNIT: AN EXPERIENCE \\ REPORT
} RECEPCIÓN CON CALIFICACIÓN DE RIESGO EN LA UNIDAD DE CUIDADOS LISTO: UN
RELATO DE EXPERIENCIA

Doi: $10.5902 / 2179769217173$

Maria Mônica Feitosa ${ }^{1}$

Iasmim Caroline Gomes Silva²

Rayane Teresa da Silva Costa ${ }^{3}$

Itaísa Cardoso Fernandes de Andrade ${ }^{4}$

Vinicius Lino de Souza Neto ${ }^{5}$

Richardson Augusto Rosendo da Silva ${ }^{6}$

RESUMO: Objetivo: relatar a experiência de uma atividade prática de estudantes de enfermagem no processo de acolhimento com classificação de risco em uma Unidade de Pronto Atendimento (UPA). Método: trata-se de um estudo descritivo, do tipo relato de experiência, realizado em uma UPA, no Nordeste do Brasil, no mês de maio de 2014. Resultados: foi possível compreender o processo de trabalho do enfermeiro na unidade e desenvolver atividades de educação em saúde aos usuários quanto à dinâmica da unidade e à prática do acolhimento por classificação de risco. 0 desenvolvimento da ação se realizou de forma sistemática e humanizada, para diminuir o tempo de espera dos usuários. Entre as dificuldades encontradas estavam a superlotação, estrutura física incipiente, falta de recursos de materiais, entre outros. Conclusão: a vivência permitiu aos estudantes refletirem sobre a prática do acolhimento com classificação de risco e entender o papel do enfermeiro como agente transformador nesse processo.

Descritores: Acolhimento; Enfermagem em emergência; Socorro de urgência.

Abstract: Aim: To report the experience of a practical activity of nursing students in the host process with risk classification in an Emergency Care Unit (UPA). Method: This is a descriptive study, which follows the experience report model, carried out in an Emergency Care Unit in Northeastern Brazil, in May 2014. Results: It was possible to understand the nursing work process in the unit, and, therefore, to develop health

\footnotetext{
${ }^{1}$ Discente de Enfermagem, Bolsista de Iniciação Científica Voluntária/ PROPESQ, Universidade Federal do Rio Grande do Norte/UFRN. Natal (RN), Brasil. E-mail: monicafeitosa14@hotmail.com

${ }^{2}$ Discente de Enfermagem, Bolsista de Iniciação Científica Voluntária/ PROPESQ, Universidade Federal do Rio Grande do Norte/UFRN. Natal (RN), Brasil. E-mail: carolgomes93@hotmail.com

${ }^{3}$ Discente de Enfermagem do Curso Bacharelado em Enfermagem da Universidade Federal do Rio Grande do Norte/UFRN. Natal (RN), Brasil. E-mail: rayane.teresa@hotmail.com

${ }^{4}$ Enfermeira graduada pela Universidade Potiguar do Rio Grande do Norte/UFRN. Natal (RN), Brasil. E-mail: itaisa23@hotmail.com

${ }^{5}$ Mestre em Enfermagem pela Universidade Federal do Rio Grande do Norte - UFRN. Professor do Curso Bacharelado em Enfermagem da Universidade Federal do Rio Grande do Norte/UFRN. Natal (RN), Brasil. E-mail: vinolino@hotmail.com

${ }^{6}$ Enfermeiro. Doutor em Ciências da Saúde. Professor Adjunto III do Curso de Graduação, Mestrado Acadêmico e Doutorado em Enfermagem do Departamento de Enfermagem da Universidade Federal do Rio Grande do Norte/UFRN. Natal, Rio Grande do Norte, Brasil. Vice-líder do grupo de pesquisa Práticas Assistências e Epidemiológicas em Saúde e Enfermagem/PAESE. E-mail: rirosendo@hotmail.com
} 


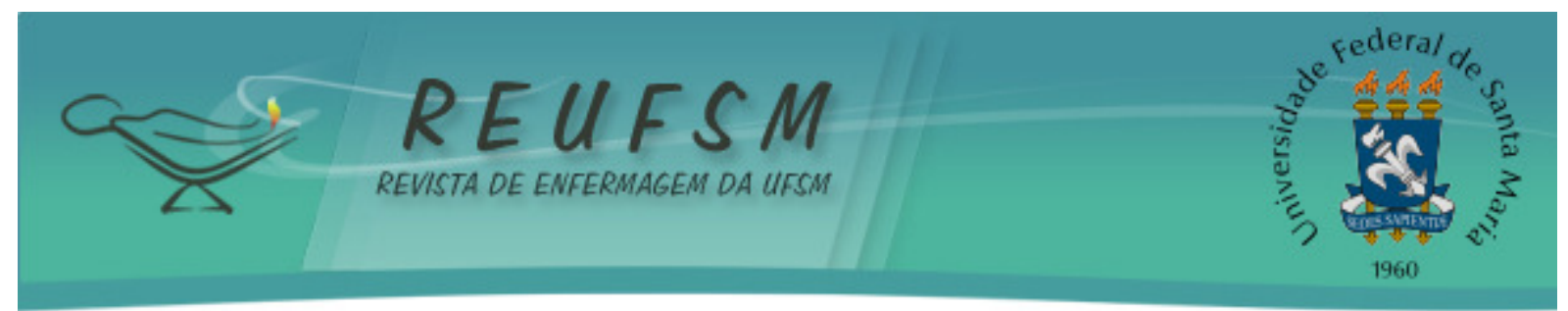

education activities to the users in relation to the dynamics of the unit and to the practice of reception by risk rating. The development of the action was carried out in a systematic and humanized manner, thus reducing the waiting time for the users. Among the difficulties encountered there were overcrowding, incipient physical structure, lack of material resources, among others. Conclusion: The experience allowed students to reflect on the practice of host with risk classification and to understand the nurse's role as an agent in this process.

Descriptors: User embracement; Emergency nursing; Emergency relief.

RESUMEN: Objetivo: Relatar la experiencia de una práctica de los estudiantes de enfermería en el proceso de acogimiento, en casos de calificación de riesgo, deuna unidad de atención de emergencia. Método: Se trata de un estudio descriptivo, el informe tipo experiencia, llevada a cabo en una unidad de atención de emergencia en el noreste de Brasil, en mayo de 2015. Resultados: Fue posible entender el proceso de trabajo de enfermería en la unidad, y desarrollar actividades de educación sanitaria a los usuarios en cuanto a la dinámica de la unidad y la práctica de la recepción por parte de la calificación de riesgo. El desarrollo de la acción se llevó a cabo de una manera sistemática y humanizado, reduciendo el tiempo de espera para los usuarios. Entre las dificultades encontradas fueron hacinamiento, la incipiente estructura física, la falta de recursos materiales, entre otros. Conclusión: La experiencia permitió a los estudiantes a reflexionar sobre la práctica de host con clasificación de riesgo y comprender el papel de la enfermera como agente en este proceso.

Descriptores: Acogimiento; Enfermería de urgencia; Socorro de urgencia.

\section{INTRODUÇÃO}

As unidades de pronto atendimento (UPA) são estruturas classificadas como de complexidade intermediária entre as unidades básicas de saúde e a unidade hospitalar, devendo funcionar $24 \mathrm{~h}$, todos os dias da semana, compondo a rede organizada de atenção às urgências e emergências no Sistema Único de Saúde (SUS). ${ }^{1}$

Com as conquistas das políticas de saúde, decorrentes da construção do SUS, alcançaram-se diversos ganhos, como por exemplo, a estruturação das políticas de saúde de urgência e emergência. Porém, ainda existem desafios que demonstram a necessidade de mudança e aperfeiçoamento do sistema, como a prática do acolhimento e o desenvolvimento da classificação de risco em alguns setores de saúde, a exemplo, na área de atenção de urgência e emergência. ${ }^{2}$

Em decorrência de um aprimoramento da qualidade do serviço, o Ministério da Saúde (MS) do Brasil, lançou, no ano de 2004, a Política Nacional de Humanização (HumanizaSUS). ${ }^{3}$ Dentre as propostas, a política no âmbito dos serviços de emergência instituiu a diretriz Acolhimento com Classificação de Risco, ou seja, realizar a prática do acolhimento de forma harmoniosa por meio da classificação de risco. ${ }^{4}$

Porém, para a sua aplicabilidade é preciso superar os seguintes desafios: a superlotação, o processo de trabalho fragmentado, conflitos e assimetrias de poder, exclusão dos usuários na porta de entrada, desrespeito aos direitos desses usuários e pouca articulação com os serviços da rede. ${ }^{4}$

Nesse sentido, erros na prática da distinção de riscos ou graus de sofrimento faz com que alguns casos se agravem na fila causando, às vezes, a morte de pessoas pela falta de atendimento em tempo hábil. Diante deste cenário, formularam-se diversos mecanismos de organização assistencial, como protocolos, para atender os usuários que 


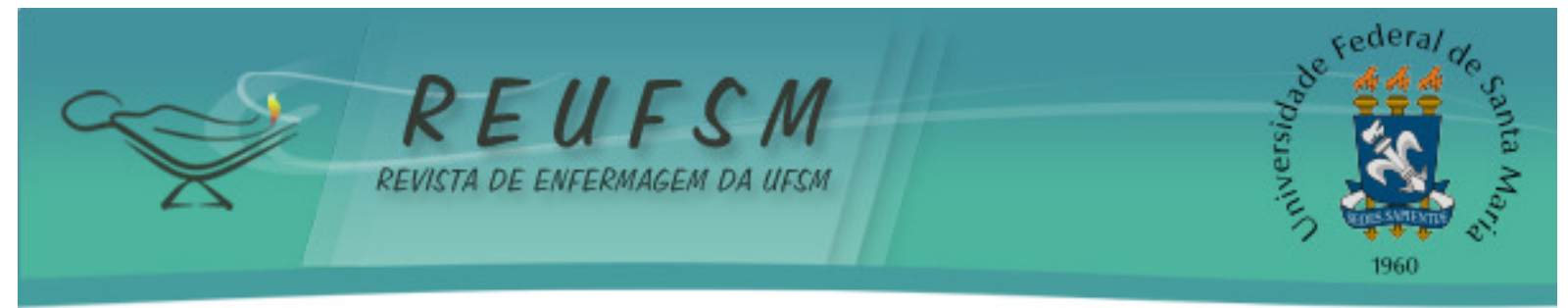

necessitam de uma conduta imediata, baseados na avaliação inicial do paciente. Assim, é possível organizar o serviço, garantindo o atendimento de todos. ${ }^{4-5}$

0 acolhimento pode ser realizado por qualquer profissional de saúde, mas cabe ao enfermeiro por meio da Consulta de Enfermagem realizar a Classificação de Risco, com base num protocolo predefinido com cores que caracterizam a gravidade ou agravamento do paciente (vermelho: emergência; amarelo: urgência; verde: pouco urgente; e azul: não urgente), o que designa a ordem do atendimento. É importante que essa organização seja divulgada com clareza para os usuários. ${ }^{5}$

Neste aspecto, a relevância do presente artigo está em explanar acerca da prática do acolhimento por meio da classificação de risco realizada por graduandos em enfermagem, bem como contribuir frente às publicações acerca da temática em âmbito nacional e internacional. Acredita-se que a experiência possa ser um incentivador para demais estudos e proporcione ao enfermeiro uma reflexão a respeito da prática, ensino, extensão, gerência e inovações das tecnologias de enfermagem.

Diante do exposto, o presente artigo tem como objetivo relatar a experiência de uma atividade prática de estudantes de enfermagem no processo de acolhimento com classificação de risco em uma UPA.

\section{METÓDO}

Trata-se de um estudo descritivo, do tipo relato de experiência, elaborado a partir das perspectivas de docentes e discentes, com base nas atividades práticas de enfermagem nos serviços de alta densidade tecnológica. Esse tipo de estudo permite a descrição de situações vivenciadas pelos autores, com a finalidade de reforçar a importância do feito na construção e remodelação dos saberes científicos e populares. ${ }^{6}$

A experiência foi compartilhada por um grupo de discentes de enfermagem do $6^{\circ}$ período e docente supervisor da Universidade Federal do Rio Grande do Norte (UFRN) no primeiro semestre do ano de 2014 , no mês de maio, com duração de seis horas diárias. A formação acadêmica do curso de enfermagem da instituição de ensino superior está distribuída em nove períodos. Assim, o projeto pedagógico do curso está estruturado a partir dos níveis de complexidade do SUS, estabelecendo-se os componentes curriculares de enfermagem com enfoque na atenção básica, média e de alta densidade tecnológica.

0 componente de ensino trabalha com base nas linhas prioritárias da rede de atenção, que são os cuidados aos agravos neurológicos, cardiovasculares, gastrintestinais, renais, respiratórios, metabólicos e de trauma, para que o discente adquira habilidades e competências relacionadas ao controle de riscos e agravos à saúde do adulto e idoso; reconhecimento do processo de trabalho do enfermeiro no contexto da alta densidade tecnológica como parte integrante dos espaços de cuidado; cuidado de enfermagem no presente serviço; diagnóstico de grupos vulneráveis, planejando e prestando cuidados de enfermagem referentes às situações de urgências, emergências e cuidados intensivos, com carga horária teórica de 220 horas e 110 horas de prática.

A carga horária prática é desenvolvida por meio do estágio curricular, e permite aos discentes vivenciar a realidade dos serviços de saúde, compartilhar saberes, trabalhar em equipe e refletir acerca da profissão. Também proporciona a estruturação do senso crítico, e estimula a prática do raciocínio sistemático, mecanismo primordial no presente setor, pois os cuidados devem ser imediatos.

Assim, as atividades práticas de urgência e emergência ocorreram na UPA, situada no distrito oeste de Natal/RN. Em sua estrutura física, conta com uma sala de classificação de risco, seis ambulatórios médicos, funcionando apenas três, decorrente da falta de profissionais, e problemas de gestão municipal. Possui também, laboratório para exames 


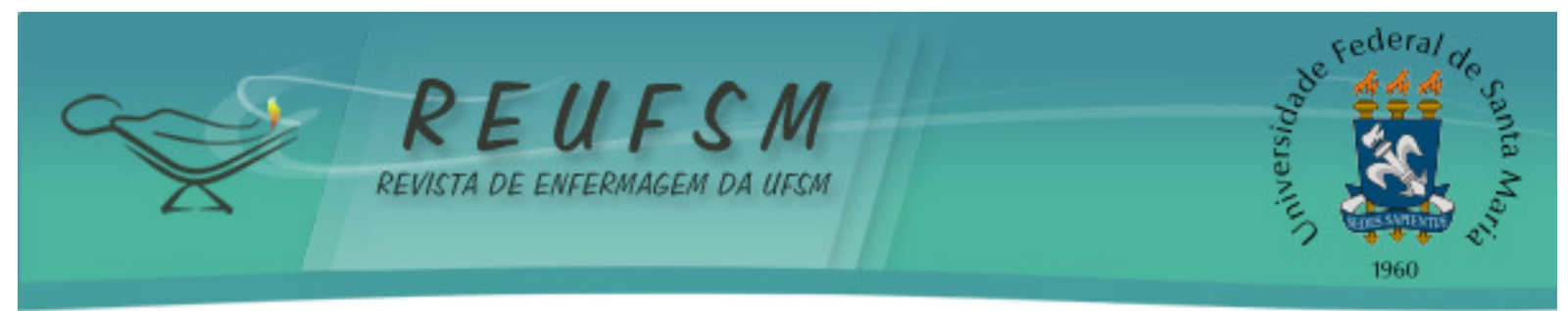

clínicos, serviços de odontologia, radiologia, pediatria, e campos de unidade de terapia intensiva, contando com 15 leitos.

A unidade conta também com 151 profissionais em atividade, sendo médicos (clínicos gerais), enfermeiros, nutricionistas, farmacêuticos, bioquímicos, assistentes sociais, técnicos de enfermagem, técnicos de laboratório, assistentes administrativos, copeiros, maqueiros e profissionais de higienização, conforme as diretrizes estabelecidas pelo MS para o funcionamento de uma UPA. ${ }^{7-8}$

\section{RESULTADOS}

A experiência compartilhada pelos discentes e docentes proporcionou momentos disciplinares, como também o dimensionamento sobre as diversas adversidades que os serviços de assistência à saúde possuem. A receptividade dos profissionais, tanto de enfermagem como de outras áreas, foi acolhedora e humana. No primeiro contato, os docentes da disciplina apresentaram todos os setores, em seguida, distribuíram os discentes de enfermagem.

0 processo de trabalho dos profissionais de enfermagem da UPA se caracteriza como fragmentado percebendo, a partir de algumas ações e intervenções realizadas, divergindo das práticas seguras e cientificas. No setor de classificação de risco, percebeuse por meio de conversas diretas com os usuários, que alguns desconheciam o setor em que estavam, a finalidade e o porquê do motivo da espera.

Decorrente da necessidade, discentes e docentes planejaram atividades de educação em saúde para serem implementadas no setor. A priori, buscou-se por estudos científicos nas bases de dados Literatura Latino-Americana e do Caribe em Ciências da Saúde (LILACS), Scientific Electronic Library Online (SCIELO) e Medical Literature Analysis and Retrieval System Online (MEDLINE), por experiências compartilhadas, como também metodologias educacionais ativas implementadas no setor. Assim, encontrou-se uma quantidade mínima, porém com informações satisfatórias frente às necessidades dos pesquisadores. Diante disso, foi estruturado um cronograma com as ações a serem desenvolvidas.

Primeiro, os discentes, realizaram uma palestra sobre a finalidade da UPA como serviço de saúde, em seguida se enfatizou a relevância da classificação de risco para os usuários do setor, demonstrando a dinâmica de atendimento da unidade, como forma de conscientizar a população. Para tanto, utilizaram banners e cartazes expostos na própria UPA, sendo elaborados folders informativos sobre a relevância da classificação de risco. Depois, os alunos orientaram os usuários que chegavam à unidade a dirigirem-se à recepção para identificação por meio do preenchimento da ficha de atendimento, para que esperassem o momento de ser encaminhada a sala de classificação de risco.

Quando o usuário entrava na sala, era chamado por seu nome e com cordialidade, com o intuito de deixá-lo à vontade, pois já se tinha em mãos sua ficha com os dados de identificação. Posteriormente, verificaram-se os sinais vitais e procedia-se à entrevista com o intuito de se identificar a queixa principal, antecedente pessoal e familiar, sinais e sintomas, motivos que os levaram ao serviço de saúde. Também se utilizava o tempo destinado à entrevista para realizar orientações voltadas para a prevenção de complicações relacionadas aos fatores de riscos identificados.

Em seguida, iniciava-se a classificação para encaminhá-los à consulta médica segundo o grau de risco. Os pacientes eram identificados por meio de pulseiras com as respectivas cores: vermelho - atendimento imediato, tempo zero; amarelo - entre 10 e 60 minutos, atendimento rápido; verde - até 120 minutos; e azul - 240 minutos ou mais. Caso 


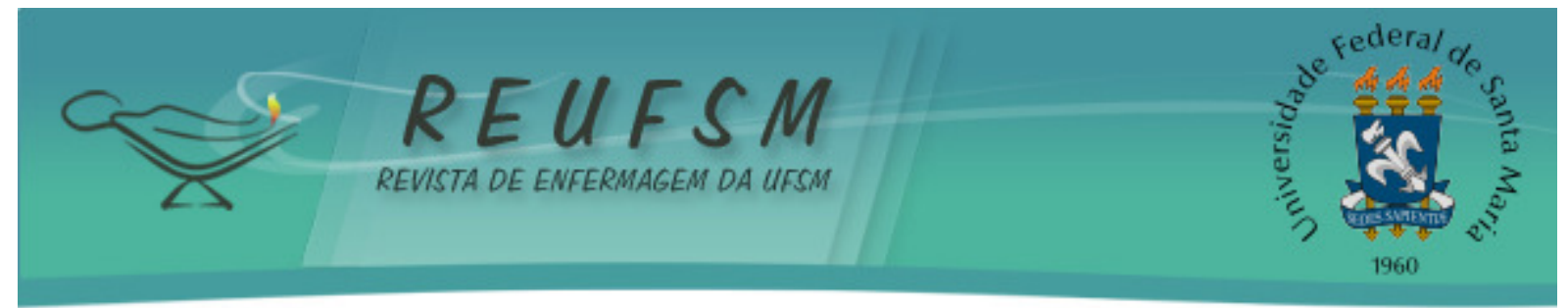

- usuário apresentasse condições clínicas descompensadas, eram encaminhados diretamente para a sala de estabilização, acompanhado pelo enfermeiro da classificação.

Apesar das ações educativas de conscientização da população sobre a classificação de risco, observaram-se dificuldades quanto à sua operacionalização, tais como: superlotação da UPA decorrente da atenção básica não atender as demandas necessárias, estrutura física deficiente, a falta de recursos de materiais, incompreensão por parte da população sobre o propósito da UPA e o tempo longo de espera para os casos onde não existia gravidade. Assim, percebendo as fragilidades, além das ações implementadas, foi proposto para os gerentes do serviço um plano de educação continuada para com os profissionais de saúde.

\section{DISCUSSÃO}

$\mathrm{Na}$ experiência compartilhada pelos discentes percebeu-se que o acolhimento se caracteriza como um processo contínuo que envolve sensibilidade e conhecimento técnicocientífico dos profissionais para identificar necessidades de saúde derivadas de processos sociais, físico-biológicos, mentais e ambientais. Esse processo permite a identificação de informações a respeito da saúde do usuário assim como o perfil da população atendida, porém requer dos profissionais de saúde habilidades e competências. ${ }^{9}$

Por isso, os enfermeiros devem deter conhecimento sobre as condições clínicas, cirúrgicas, psicossociais, para que assim, relacionem os conhecimentos teóricos e práticos, como também noções de políticas públicas de saúde que direcionam aos serviços de referências e contrarreferência e a ambiência do trabalho. Os setores de pronto atendimento facilitam essa interação, requerendo decisões imediatas. Porém, a classificação de risco, possibilita ao enfermeiro relações conflituosas, como por exemplo, a não concordância do usuário quanto a prioridade de atendimento. ${ }^{10-11}$

Assim, na experiência compartilhada pelos discentes em alguns momentos percebeu-se que os usuários não ficavam satisfeitos, pois queriam ser atendidos imediatamente, e não apresentavam prioridade imediatas. Estudo pontua que nos serviços de classificação de risco, os enfermeiros estão sobre forte pressão, pois precisam ter uma decisão imediata e sofrem ,em alguns momentos, ameaças dos usuários, gerando assim um ambiente inseguro. ${ }^{12-13}$

Assim, que as práticas de educação em saúde tem relevância no setor, para que os usuários possam entender a dinâmica do atendimento, e respeitar o processo de trabalho dos profissionais do serviço.

Além disso, percebeu-se com a experiência compartilhada, que para operar a prática do acolhimento requer mudanças significativas no processo de trabalho do enfermeiro. Pois, acolher é uma tecnologia que profissionais de saúde devem realizar, ao qual pressupõe a garantia de acesso a todas as pessoas, como também otimiza, qualifica e reorganiza as práticas assistenciais. Porém, 0 que se visualiza são serviços que desvalorizam a prática do acolhimento, pois acreditam que essa prática esteja voltada a ampliação da ambiência, que se traduz por um atendimento rápido e um ambiente confortável. ${ }^{14}$

Contrapondo essa ideia, estudo aponta que a prática do acolhimento proporciona agilidade no atendimento, priorizando os usuários que necessitam de atenção imediata, organizando os serviços, o fluxo do usuário e a intervenção de forma eficaz, evitando sequelas. ${ }^{15}$ Além disso, pesquisadores pontuam que a prática do acolhimento na classificação de risco transversaliza os saberes da ciência e proporciona segurança ao paciente. ${ }^{16}$ 


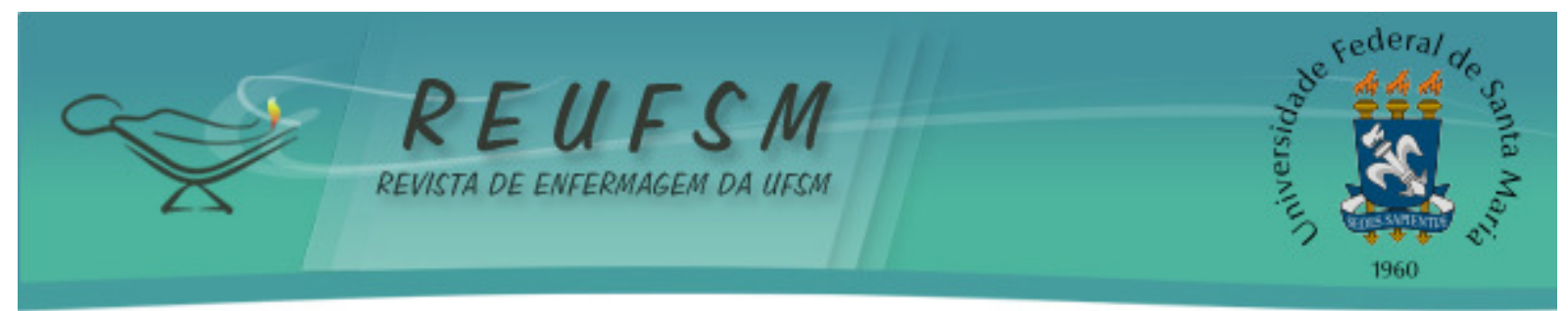

Outro ponto presente na experiência dos discentes foi que, além das dificuldades colocadas sobre a praticidade do acolhimento, algumas se fizeram pertinentes como a falta de materiais necessários para realizar a classificação de risco. Outro aspecto, a superlotação decorre de diversos fatores como, por exemplo serviços de atenção básica não funciona da forma que deveria. Essas dificuldades foram inerentes em outras realidades, como pontua estudo realizado por pesquisadores da Universidade Federal do Ceara que aponta além desses entraves, outros desafios colocados, como a dificuldade na manutenção do fluxo de atendimento correto, sobrecarga de trabalho e impasses na utilização do protocolo classificador. ${ }^{16-17}$

Percebeu-se que, na prática, a utilização dos protocolos sofria algumas modificações decorrentes da necessidade de adaptarem-se as queixas dos usuários. 0 estudo pontua que o uso do protocolo na classificação de risco corrobora para a instrumentalização, orientação e otimização das decisões dos profissionais da saúde. ${ }^{18}$

Para tanto, os enfermeiros devem buscar continuadamente por conhecimento, seja especializado, ou generalizado, com o objetivo de elaborar uma assistência com base nas necessidades sociais e biológicas dos usuários. Para isso, a prática da educação continuada vem ganhando espaços, seja na atenção primaria à saúde, como também no âmbito hospitalar. Nota-se que gestores de serviços de saúde estão cada vez mais empenhados em elaborar e implementar núcleos de educação para os profissionais do serviço.

A educação continuada caracteriza-se por ser essencial nos setores da saúde, pois contribui para o desenvolvimento da competência profissional, levando a aquisição de novos conhecimentos da realidade. ${ }^{19}$ Assim, para que se torne efetiva, deve fazer parte do planejamento da instituição, como uma ferramenta estratégica para o desenvolvimento de pessoas e ser planejada considerando as necessidades, de forma individual ou institucional e avaliada sistematicamente. Devem ser utilizadas metodologias que visem identificar problemas, a partir da observação da realidade pelo sujeito para então propor soluções. ${ }^{20}$

\section{CONSIDERAÇÕES FINAIS}

Esta experiência oportunizou aos discentes e docentes de enfermagem refletir sobre a prática do acolhimento, como também dimensionar os saberes sobre a política nacional de humanização. Além disso, os momentos levaram a aproximação dos discentes da realidade dos serviços de saúde, para que assim pudessem sobre as necessidades dos usuários e otimizar no processo de ensino e aprendizagem .

Acredita-se que a prática da classificação de risco e acolhimento tem a finalidade de reorganizar o fluxo de atendimento, permitindo distinguir os casos urgentes dos não urgentes, agilizando o serviço, diminuindo o tempo de espera para a consulta médica, permitindo, assim, visualizar a carência de informações dos usuários frente à classificação.

Compreendeu-se que a educação continuada é uma ferramenta fundamental que atenua as fragilidades na formação, qualificando os serviços prestados por instituições de saúde. Além disso, notou-se que o enfermeiro é ator principal frente às mudanças na assistência, pois as práticas de educação em saúde remodelam os saberes e ampliam os horizontes, seja do usuário, como dos profissionais.

\section{REFERÊNCIAS}

1. Brasil. Ministério da Saúde. Secretaria de Atenção a Saúde. Departamento de Atenção Especializada. Manual instrutivo da rede da atenção as urgências e emergências no Sistema Único de Saúde (SUS). Brasília: Editora do Ministério da Saúde; 2013 [acesso em 2014 jul 25]. 86 p. Disponível em: 


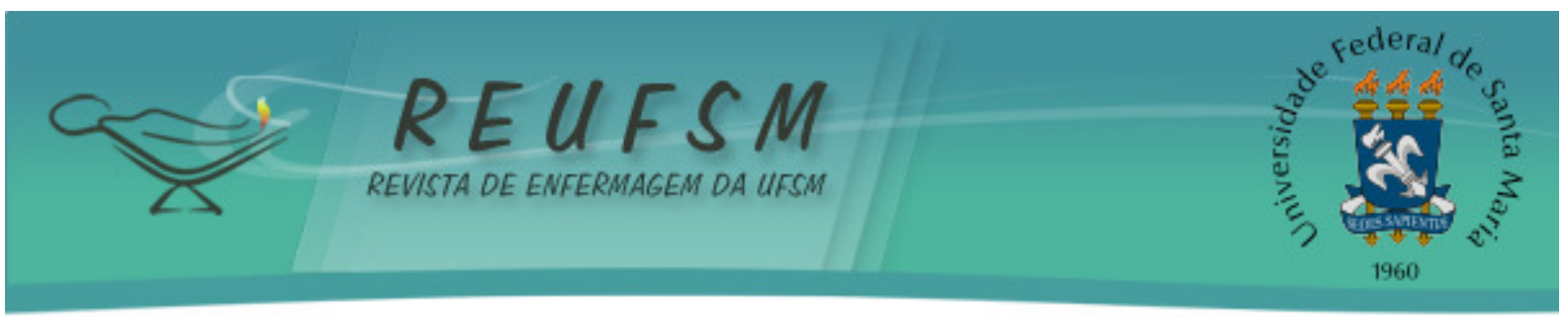

http://bvsms.saude.gov.br/bvs/publicacoes/manual_instrutivo_rede_atencao_urgenci as.pdf.

2. Brasil. Ministério da Saúde. Secretaria de Atenção à Saúde. Política Nacional de Humanização da atenção e Gestão do SUS. Acolhimento e classificação de risco nos serviços de urgência. Brasília: Ministério da Saúde; 2009 [acesso em 2014 jul 25]. 56 p. Disponível em: http://bvsms.saude.gov.br/bvs/publicacoes/acolhimento_classificacao_risco_servicos_2009.p df. (Série B. Textos Básicos de Saúde).

3. Brasil. Ministério da Saúde. Secretaria Executiva. Núcleo técnico da Política Nacional de Humanização. Humaniza SUS: Política Nacional de Humanização: a humanização como eixo norteador das práticas de atenção e gestão em todas as instâncias do SUS. Brasília: Ministério da Saúde; 2004 [acesso em 2014 jul 25]. 20 p. Disponível em: http://bvsms.saude.gov.br/bvs/publicacoes/humanizasus_2004.pdf.

4. Rossaneis MA, Haddad MCL, Vannuchi MTO, Sentone ADD, Gabriel CS. Acolhimento com avaliação e classificação de risco em hospital público de médio porte e a responsabilidade da atenção básica. UNOPAR Cient Ciênc Biol Saude [Internet]. 2011jun [acesso em 2014 jul 25];13(3)169-74. Disponível em: http://www.pgsskroton.com.br/seer/index.php/JHealthSci/article/view/1191/1144.

5. Rodrigues APB, Ribeiro SCL, Santos AMR, Moura MEB, Mesquita GV, Brito JNPO. Concepção da equipe de enfermagem sobre acolhimento com classificação de risco. Rev Enferm UFPE on line [Internet]. 2014 ago [acesso em 2014 jul 25];8(8):2626-32. Disponível em: http://www.revista.ufpe.br/revistaenfermagem/index.php/revista/article/download/6619/9955.

6. Minayo, CS. Pesquisa social: teoria, método e criatividade. $26^{\mathrm{a}}$ ed. Petrópolis: Vozes; 2007.

7. Brasil. Ministério da Saúde. Portaria $n^{\circ} 1.601$, de 7 de julho de 2011. Estabelece diretrizes para a implantação do componente Unidades de Pronto Atendimento (UPA 24h) e o conjunto de serviços de urgência 24 horas da Rede de Atenção às Urgências, em conformidade com a Política Nacional de Atenção às Urgências. Diário Oficial da União, Brasília; 2011 jul 8. Seção 1; p. 70-2.

8. Prefeitura Municipal do Natal. UPA Cidade da Esperança tem nova ambulância e vai atender pediatria. Natal; 2014 [acesso em 2014 jul 20]. Disponível em: http://www.natal.rn.gov.br/noticia/ntc-18995.html.

9. Guedes MVC, Henriques ACP T, Lima MMN. Acolhimento em um serviço de emergência: percepção dos usuários. Rev Bras Enferm [Internet]. 2013 jan/fev [acesso em 2014 jul 25]; 66(1):31-7. Disponível em: http://www.scielo.br/pdf/reben/v66n1/v66n1a05.pdf.

10. Gehlen GC, Lima MADS. Nursing work in care practice at emergency care units in Porto Alegre/ RS. Invest Educ Enferm. 2013;31(1):26-35.

11. Nascimento ERP, Hilsendeger BR, Neth C, Belaver GM, Bertoncello KCG. Classificação de risco na emergência: avaliação da equipe de enfermagem. Rev Enferm UERJ [Internet]. 2011 jan/mar [acesso em 2014 jul 25];19(1):84-8. Disponível em: http://www.facenf.uerj.br/v19n1/v19n1a14.pdf.

12. Foley A, Durant J. Let's ask that out front: health and safety screenings in triage. J Emerg Nurs. 2011;37(5):515-6.

13. Nascimento ERP, Hilsendeger BR, Neth C, Belaver GM, Bertoncello KCG. Acolhimento com classificação de risco: avaliação dos profissionais de enfermagem de um serviço de 


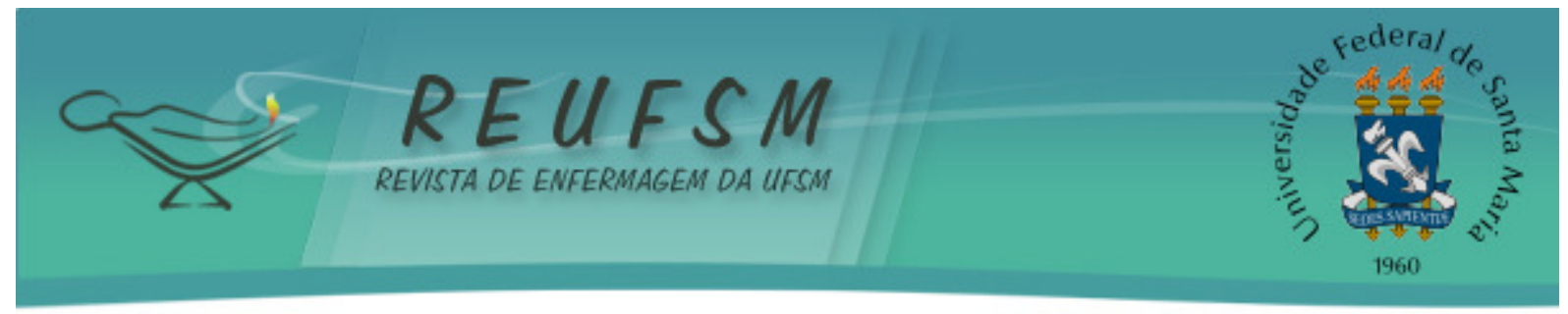

emergência. Rev Eletrônica Enferm [Internet]. 2011 out/dez [acesso em 2014 jul 25];14(3):597-603. Disponível

http://www.fen.ufg.br/revista/v13/n4/pdf/v13n4a02.pdf.

14. Nonnenmacher CL, Weiller TH, Oliveira SG. Opiniões de usuários de saúde sobre o acolhimento com classificação de risco. Rev Eletrônica Enferm [Internet]. 2012 jul/sep [acesso em 2014 jul 25];14(3):541-9. Disponível em: http://www.fen.ufg.br/revista/v14/n3/pdf/v14n3a10.pdf.

15. Duro CLM, Lima MADS, Levandovski PF, Bohn MLS, Abreu KP. Percepção de enfermeiros sobre a classificação de risco em unidades de pronto atendimento. Rev RENE [Internet]. 2014 maio/jun [acesso em 2014 jul 25];15(3):447-54. Disponível em: http: / / www.revistarene.ufc.br/revista/index.php/revista/article/view/1551/pdf.

16. Goyanna NF, Netto JJM, Freitas CASL, Ponte MAC, Dias MSA. Acolhimento com avaliação e classificação de risco: dos desafios às potencialidades. Sanare (Sobral, Online). [Internet] 2014 jan/jun [acesso em 2014 jul 25];13(1):119-24. Disponível em: http://sanare.emnuvens.com.br/sanare/article/view/442/296.

17. Guedes MVC, Henriques ACPT, Lima MMN. Acolhimento em um serviço de emergência: percepção dos usuários. Rev Bras Enferm. 2013;66(1):31-7.

18. Pinto Júnior D, Salgado PO, Chianca TCM. Validade preditiva do Protocolo de Classificação de Risco de Manchester: avaliação da evolução dos pacientes admitidos em um pronto atendimento. Rev Latinoam Enferm. 2012;20(6): 235-9.

19. Caveião C, Hey AP, Montezeli JH, Barros APMM, Sordi JA, Santos SC. Desafios ao Enfermeiro na Implantação da Classificação de Risco em Unidade Mista. Rev Enferm UFSM [Internet]. 2014 jan/mar [acesso em 2014 l 25];4(1):189-96. Disponível em: http://cascavel.ufsm.br/revistas/ojs-2.2.2/index.php/reufsm/article/viewFile/10527/pdf.

20. Paulino VCP, Bezerra ALQ, Branquinho NCSS, Paranaguá TTB. Ações de educação permanente no contexto da estratégia saúde da família. Rev Enferm UERJ. 2012;20(3):312-6.

Data de recebimento: 07/03/2015

Data de aceite: 30/01/2017

Endereço do autor: Vinicius Lino de Souza Neto

Universidade Federal do Rio Grande do Norte, Centro de Ciências da Saúde - Departamento de Enfermagem Campus Central, s/n, Lagoa Nova 59078-970 Natal - RN E-

mail:vinolino@hotmail.com 